\title{
High-resolution transparent x-ray beam location and imaging
}

\author{
Roelof van Silfhout, ${ }^{1, *}$ Anton Kachatkou, ${ }^{1}$ Nicholas Kyele, ${ }^{1}$ Peter Scott, ${ }^{1}$ Thierry Martin,, ${ }^{2}$ and Sergey Nikitenko ${ }^{3}$ \\ ${ }^{1}$ The University of Manchester, Sackville Street, Manchester, M13 9PL, United Kingdom \\ ${ }^{2}$ European Synchrotron Radiation Facility, BP 220, F-38043 Grenoble, France \\ ${ }^{3}$ DUBBLE Collaborative Research Group at European Synchrotron Radiation Facility , BP 220, F-38043 Grenoble, France \\ *Corresponding author: r.vansilfhout@manchester.ac.uk
}

Received November 8, 2010; revised December 16, 2010; accepted January 3, 2011; posted January 11, 2011 (Doc. ID 137767); published February 15, 2011

\begin{abstract}
We present a high-resolution in situ imaging and localization method of energetic particle beams. Recording of the scattered radiation from a thin featureless foil, placed in the path of the beam, and taken with a pinhole or coded aperture camera arrangement magnifies beam movements at the sensor. At the same time, a magnified image of the beam is available with an exceptional signal-to-noise ratio. We show measurement results of the level of precision that can be achieved and compare them to theoretical limits based on the signal-to-noise levels. @ 2011 Optical Society of America

OCIS codes: $120.3940,040.7480$.
\end{abstract}

X-ray beams produced by third generation synchrotron light sources are unique tools in the nondestructive probing and imaging of matter on an atomic scale for a wide range of scientific and industrial research. Advances in source and focusing optics have enabled researchers to routinely conduct experiments on microscopic samples. Colocating these microfocused beams with samples and focusing the beams optimally becomes a challenging task that requires nonevasive or transparent x-ray beam imaging sensors with high spatial resolution.

Several types of transparent beam position sensors have been developed, tested, and implemented on beam lines at synchrotrons worldwide. These devices rely on fluorescence, photoemission, photoconduction, or photoluminescence. The most common device records merely a center of gravity beam position by recording fluorescence signals from a thin metal foil [1]. These devices have several disadvantages. To cover a wide energy range, several types of thin metal foils are needed that create anomalies near the absorption edges that will affect spectroscopic measurements. Photoemissive devices measure photoelectrons from blades placed into the tails of the beam [2]. Photoluminescence devices monitor the visible luminescent light emitted by a chamber containing a gas (e.g., He, $\mathrm{N}_{2}$ ) placed in the path of the beam [3]. Semiconductor photodiodes have been used that feature a small central hole leaving the center of the beam undisturbed [4,5]. More recently, center of gravity x-ray beam sensors based on thin silicon and diamond diodes have been reported [6,7]. Other methods reported in the literature include modified ion chambers [8]. All of these methods suffer from limited linear range and apparent beam motion on small changes in beam shape.

In this Letter, we present a method that images the radiation scattered by a thin low-density foil using a pinhole or coded aperture. Such a scheme is attractive for several reasons $[9,10]$. First, the detector is placed well away from the direct beam and has a line of sight that is at a right angle with the incident beam. Second, the scatter foil absorbs a negligible amount of radiation and does not introduce any absorption edges in the hard x-ray part of the spectrum. Third, the scatter geometry allows for magnification $(L / D)$ of beam displacements at the detector (see Fig. 1). The positional sensitivity of the device for beam displacements along the $x$ and $y$ directions may be further increased by tilting the sensor.

Kapton foils are a good choice, because above $3 \AA^{-1}$ its scatter curve is smooth and featureless. Thus, in our detection scheme, the scatter yield correction is only weakly energy dependent above $5 \mathrm{keV}$, allowing for

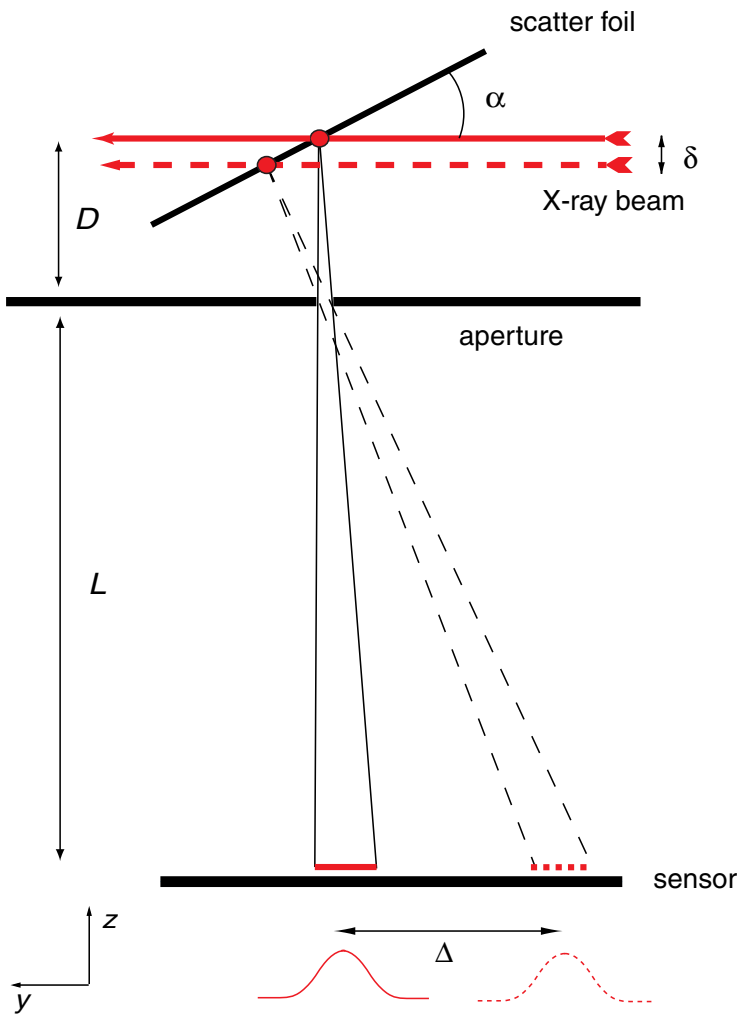

Fig. 1. (Color online) Schematic of the beam imaging instrument (not drawn to scale). The x-ray beam hits a foil tilted at an angle $\alpha$ with respect to the incident beam. Scattered radiation passes through a pinhole and is recorded by an x-ray imaging sensor positioned below the pinhole. Any beam displacements are magnified at the sensor. 
absolute photon flux measurements across a wide range of beam energies.

Let $x^{\prime}$ and $y^{\prime}$ describe the plane of the detector, $\delta x$ and $\delta z$ are beam displacements in the $x$ - and $z$-directions from the midposition, respectively. If we consider the thickness of the pinhole sheet to be negligible and the size of the footprint of the beam to be small we have

$$
\begin{gathered}
\frac{\Delta x^{\prime}}{\delta x}=\frac{L}{D}, \\
\frac{\Delta y^{\prime}}{\delta z}=\frac{L}{D} \frac{1}{\tan \alpha} \frac{1}{(1+\delta z / D)},
\end{gathered}
$$

where $\Delta x^{\prime}$ and $\Delta y^{\prime}$ are the measured shift at the detector, $L$ the detector to pinhole and $D$ the pinhole to scatter foil distance. The foil tilt angle is denoted by $\alpha$. The third term on the right-hand side of Eq. (2) is a correction for the variation in distance between the footprint of the beam and the pinhole. This term can be neglected for small (compared to $D$ ) beam movements. We now estimate the expected resolution of beam displacement measurements of our technique along the horizontal $\left(\sigma_{\delta x}\right)$ and vertical $\left(\sigma_{\delta z}\right)$ directions using error propagation rules. For given experimental conditions, the resolution in displacement measurements at the detector $\left(\sigma_{x}\right.$ and $\left.\sigma_{y}\right)$ are calculated using a method outlined by Bobroff [11]. Their values depend on the signal-to-noise ratio ( $\overline{\mathrm{SN}})$ and number of sample points, which depend on the pixel size and the size of the footprint of the beam on the sensor. A higher SNR and more sampled points improve the position resolution.

The relation between the measured $\sigma_{x}$ and $\sigma_{y}$ and the corresponding $\sigma_{\delta x}$ and $\sigma_{\delta z}$ is given by error propagation rules applied to Eqs. (1) and (2). For quantitative results of this analysis, we refer to Fig. 2 , where we have plotted the results of calculations for expected resolution in the $z$-coordinate performed using measured values for sensor background, maximum pixel intensity, sensor shot, and read-out noise and beam size for the ID32 beamline at the European Synchrotron Radiation Facility (ESRF) assuming a Gaussian beam profile.

The solid curve denotes the calculated results if we keep the integration time constant, whereas the dashed curve represent the case in which the peak profile intensity is kept constant (by increasing integration time) as the magnification is increased. As the magnification is increased (increasing $L$ while leaving $D$ constant), the resolution increases initially until the loss of SNR (due to

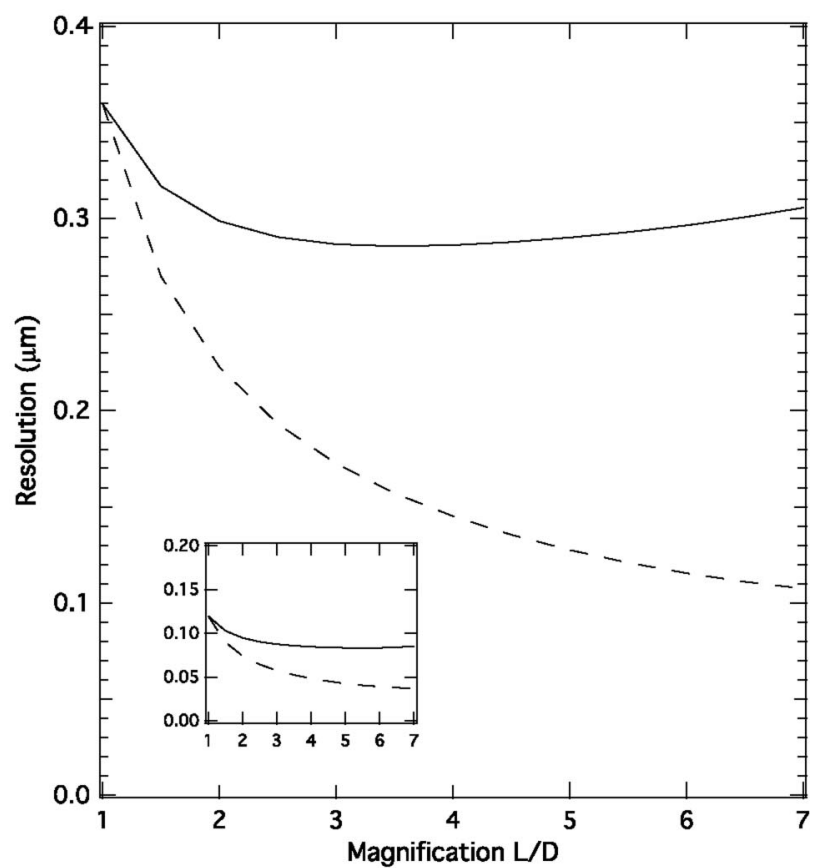

Fig. 2. Calculations (solid curve) for expected resolution of the setup in measuring the actual beam displacement using $\alpha=24^{\circ}$. Other model parameters: maximum pixel intensity for $L / D=2$ is 150 arbitrary units (abu), image background level is $2.0 \mathrm{abu}$, pinhole diameter $500 \mu \mathrm{m}$, and read-out noise $\sigma_{r}=1.74 \mathrm{abu}$. The inset shows the same calculations for maximum pixel intensity of $500 \mathrm{abu}$. The dashed curve shows the same result for the case where the peak intensity of the profiles is kept constant.

spreading of scattered intensity over a larger sensor area) overcomes the benefit of more sampled points over the intensity distribution at the detector. To demonstrate the effect of SNR, we have also calculated (see inset in Fig. 2) the predicted resolution for ID32 at a higher machine current of $300 \mathrm{~mA}$, leaving all other parameters unchanged.

Table 1 shows a detailed comparison between the model predictions and measurements performed at three ESRF beamlines (ID6, ID32, and BM26A) with different beam energies and integrated intensities.

The data were collected using an embedded system that features programmable hardware for real-time processing of images taken by a CMOS image sensor that was optically coupled to a P43 scintillator $[12,13]$. Tasks such as background subtraction, row/column pixel summing, and data compression are performed at real time.

\begin{tabular}{|c|c|c|c|c|c|c|}
\hline $\begin{array}{l}\text { ESRF Beam } \\
\text { Line }\end{array}$ & $\begin{array}{c}\text { Beam Size } \\
(H \times V)(\mu \mathrm{m})\end{array}$ & $\begin{array}{c}\text { Energy } \\
(\mathrm{keV})\end{array}$ & $\begin{array}{c}\text { Magnification } \\
L / D\end{array}$ & $\begin{array}{l}\text { Pinhole } \\
\text { Diameter } \\
(\mu \mathrm{m})\end{array}$ & $\begin{array}{c}\text { Calculated } \\
\text { Resolution } \\
\sigma_{\delta z}(\mu \mathrm{m})\end{array}$ & $\begin{array}{c}\text { Measured } \\
\text { Resolution } \\
\tilde{\sigma}_{\delta z}(\mu \mathrm{m})\end{array}$ \\
\hline ID6 (200 mA) & $\begin{array}{c}480 \times 170 \\
\text { unfocused }\end{array}$ & $20 \mathrm{keV}$ & 3 & 300 & 0.85 & $0.80(3)$ \\
\hline ID32 (90 mA) & $300 \times 40$ focused & $16 \mathrm{keV}$ & 7 & 500 & 0.3 & $0.40(8)$ \\
\hline $\begin{array}{l}\text { BM26A } \\
(200 \mathrm{~mA})\end{array}$ & $300 \times 300$ focused & $12 \mathrm{keV}$ & 2 & 500 & 1.6 & $1.8(1)$ \\
\hline
\end{tabular}

Table 1. Comparison of Calculated and Measured Resolution ${ }^{a}$

\footnotetext{
${ }^{a}$ Comparison of expected $z$-position measurement resolution, $\sigma_{\delta z}$ with measurements performed at three different ESRF beamlines each with different energy and focusing conditions: ID6 cryo-undulator, unfocused beam size defined by slits; ID32 undulator, focused with compound refractive lenses; and BM26A bending magnet, focused with a combination of mirror and sagittal focusing monochromator.
} 

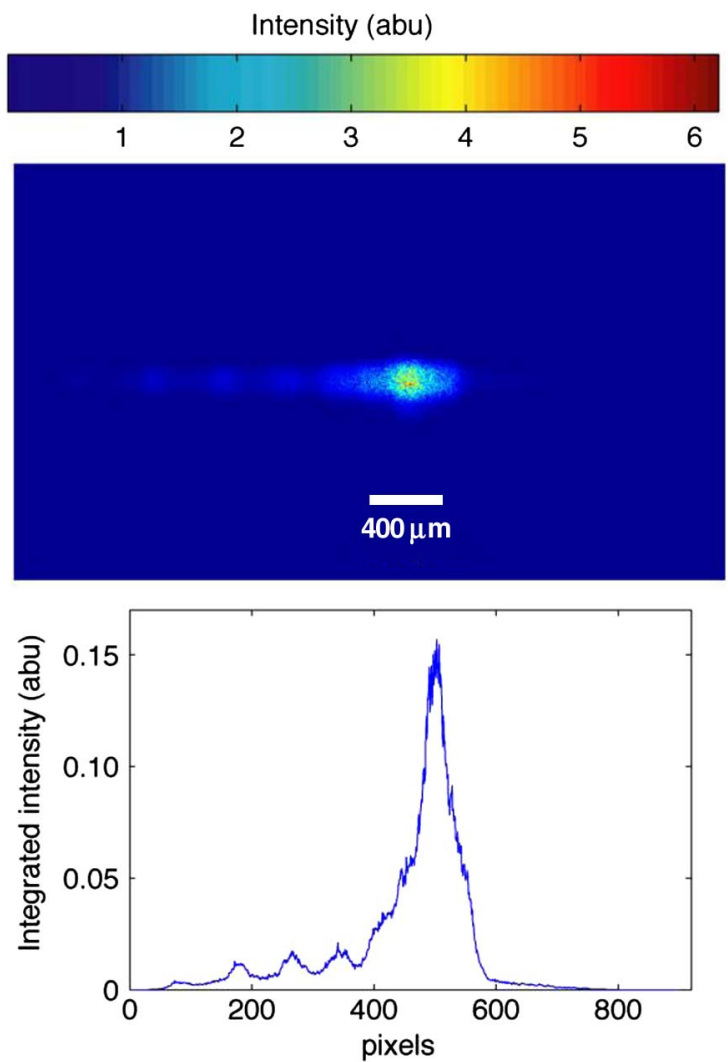

Fig. 3. (Color online) False color x-ray beam image $(x-z$ plane) taken using a cross-shaped aperture (upper frame). The image shows the deconvolution of the measured data taken at a focused bending magnet beamline BM26A and the known laser cut cross-shaped aperture. The lower frame shows the integrated horizontal beam profile.

To remove the effect of beam movements during the measurements we used two procedures. For the relatively large, unfocused beam, a fixed slit of given (smaller) size was inserted close to our device, hence making the transmitted beam appear stable and the rms value of the measured beam position would give a good estimate of the precision of our device. For the measurements with a focused beam, the use of a small slit was impractical and we therefore used the rms value of the data obtained by subtracting the moving average of the measured data from the unprocessed data as a measure for resolution. The actual number of points used in the averaging was determined experimentally by increasing it until a saturation of the rms value was evident.

From a beam position resolution point of view, it makes little sense to choose a very small pinhole. However, for beam imaging a small pinhole is imperative because the achievable resolution is set by the size of the pinhole. The use of multiple or coded apertures has been tested with the aim to increase detected signal levels. Unfortunately, the required image processing for unfolding the pinhole pattern from the measured image can become prohibitive if fast updates of beam position are required. We have therefore opted to use an aperture that consists of two orthogonal and crossed $100 \mu \mathrm{m}$ wide and $3 \mathrm{~mm}$ long slits ( + shaped aperture). This type of aperture provides a good imaging resolution after a simple deconvolution and at the same time enables better SNR values for the beam profiles that are measured simply by adding rows and columns, respectively.

Figure 3 shows the excellent imaging performance as achieved with $L / D=2$ at a relatively low-flux, bendingmagnet beamline that had issues with a ribbed, sagittally focused monochromator causing multiple foci [14]. With this type of coded aperture, gains of more than 1 order of magnitude in SNR are easily achieved with the prospect of $i n$ situ position monitoring with a resolution of several nanometers, which is smaller than the best focal spots than have been achieved.

In summary, we have shown a simple, robust and precise way of fully characterizing intense, x-ray beams transparently enabling a controlled and precise delivery of probing beams onto microscopic samples. The presented method works well for a wide range of beam intensities, such as found at bending magnet or insertion devices.

Unlike any other in situ method, our method provides both highly precise beam position measurements and images of the impinging beam providing a new level of diagnostics. A similar scheme could easily be applied to beams of other particles. We have provided a detailed comparison of the achieved resolution with a simple theoretical model that shows that the resolution is fully understood in terms of detector noise and achieved SNR.

The authors gratefully acknowledge Drs. B. Detlefs, C. Detlefs, and D. Pothin and the ID6, ID32, and BM26 staff for their support during our experiments at the ESRF.

\section{References}

1. R. W. Alkire, G. Rosenbaum, and G. Evans, J. Synchrotron Radiat. 7, 61 (2000).

2. J. R. Chen, T. S. Ueng, G. Y. Hsiung, G. Y. Lin, C. T. Lee, S. L. Tsai, and S. L. Chang, J. Synchrotron Radiat. 5, 621 (1998).

3. P. Revesz and J. A. White, Nucl. Instrum. Methods Phys. Res., Sect. A 540, 470 (2005).

4. S. H. Southworth and P. L. Cowan, Nucl. Instrum. Methods Phys. Res., Sect. A319, 51 (1992).

5. R. G. van Silfhout, Nucl. Instrum. Methods Phys. Res., Sect. A 403, 153 (1998).

6. M. R. Fuchs, K. Holldack, M. Bullough, S. Walsh, C. Wilburn, A. Erko, F. Schafers, and U. Mueller, Rev. Sci. Instrum. 79, 063103 (2008).

7. P. Bergonzo, D. Tromson, and C. Mer, J. Synchrotron Radiat. 13, 151 (2006).

8. A. N. Artemiev, S. Latushkin, V. Mikhailov, V. Reznov, and L. Yudin, Nucl. Instrum. Methods Phys. Res., Sect. A477, 335 (2002).

9. R. G. van Silfhout, J. Synchrotron Radiat.6, 1071 (1999).

10. N. R. Kyele, R. G. van Silfhout, S. Manolopoulos, and S. Nikitenko, J. Appl. Phys. 101, 064901 (2007).

11. N. Bobroff, Rev. Sci. Instrum. 57, 1152 (1986).

12. R. G. van Silfhout and A. S. Kachatkou, Nucl Instrun Methods 597, 266 (2008).

13. P. F. I. Scott, A. S. Kachatkou, N. R. Kyele, and R. G. van Silfhout, Opt. Eng. 48, 073601 (2009).

14. S. Nikitenko, A. M. Beale, A. M. J. van der Eerden, S. D. M. Jacques, O. Leynaud, M. G. O'Brien, D. Detollenaere, R. Kaptein, B. M. Weckhuysen, and W. Bras, J. Synchrotron Radiat. 15, 632 (2008). 